\title{
A Case of Solid Pseudopapillary Neoplasm of the Pancreas Presenting with Left-sided Extrahepatic Portal Hypertension
}

\author{
Shinichi Nakamura ${ }^{1}$, Yukiko Takayama ${ }^{1}$, Yuko Kuboki ${ }^{1}$, Hiromi Haruyama ${ }^{1}$, \\ Maiko Kishino ${ }^{1}$, Hiroyuki Konishi ${ }^{1}$, Takashi Hatori ${ }^{1}$, Toru Furukawa ${ }^{2}$ and Keiko Shiratori ${ }^{1}$
}

\begin{abstract}
A 53-year-old woman was referred to our hospital for management of gastric varices that ran transversely across the greater curvature of the gastric body, detected during routine upper gastrointestinal endoscopy. CT identified a low-density calcified mass near the tail of the pancreas and the splenic hilum. Based on the results of radiographic and pathological investigations, the tumor was diagnosed as solid pseudopapillary neoplasm (SPN), and the gastric varices were considered to have developed secondary to occlusion of the splenic vein by the tumor mass. This is a rare case of SPN associated with splenic vein occlusion and left-sided extrahepatic portal hypertension.
\end{abstract}

Key words: gastric varices, left-sided extrahepatic portal hypertension, pancreas neoplasm, solid pseudopapillary neoplasm

(Inter Med 49: 1749-1753, 2010)

(DOI: 10.2169/internalmedicine.49.3676)

\section{Introduction}

Like esophageal varices, gastric varices are collateral veins that develop due to portal hypertension in patients with chronic liver disease, especially cirrhosis. Gastric varices also occur sometimes in patients with pancreatic disease. In particular, stenosis or occlusion of the splenic vein, caused by the spread of inflammation from chronic pancreatitis or the invasion of pancreatic cancer, sometimes triggers left-sided extrahepatic portal hypertension, associated with characteristic gastric varices extending from the fornix to the greater curvature of the gastric body (1-6). With respect to the hemodynamics, splenic vein occlusion leads to the development of a collateral pathway through which blood flows retrogradely in the short gastric veins, via the vessels of the gastric wall, and hepatopetally into the portal vein from the left gastric vein. The dilated vessels in the gastric wall can be recognized as varices.

Although inflammatory and malignant lesions of the pan- creas are the two major etiologies as discussed above, gastric varices caused by benign neoplasms of the pancreas have rarely been described in the literature, especially solid pseudopapillary neoplasm (SPN) (6-8). Here we report our experience with a patient who had SPN of the pancreas that was detected following the identification of gastric varices on the greater curvature of the gastric body.

\section{Case Report}

A 53-year-old woman was referred to our hospital for further management of suspected gastric varices based on the finding of a protruding lesion on the mucosal surface of the greater curvature of the gastric body detected by upper gastrointestinal (GI) endoscopy on a routine check-up. She was asymptomatic with no physical signs or abnormalities. Biochemical tests showed no abnormalities, and tumor markers were normal (CA19-9: <10 U/mL, DU-PAN-2: $25 \mathrm{U} / \mathrm{mL}$ ). Upper GI endoscopy revealed a submucosal tumor-like elevation that ran transversely across the greater curvature of

${ }^{1}$ Institute of Gastroenterology, Tokyo Women's Medical University, Tokyo and ${ }^{2}$ Institute of Integrated Medical Sciences, Tokyo Women's Medical University, Tokyo

Received for publication March 19, 2010; Accepted for publication April 26, 2010

Correspondence to Dr. Shinichi Nakamura, shin.n@d5.dion.ne.jp 


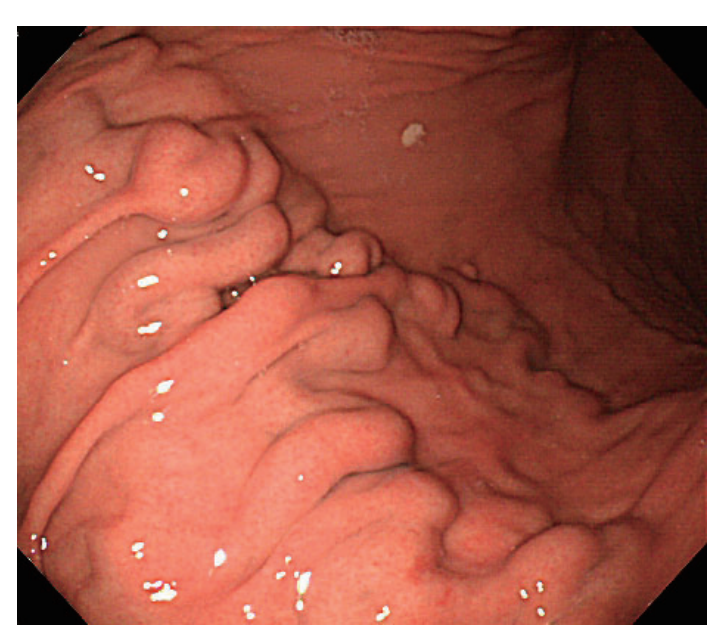

Figure 1. Endoscopic view showing tortuous varices running transversely across the greater curvature of the gastric body.

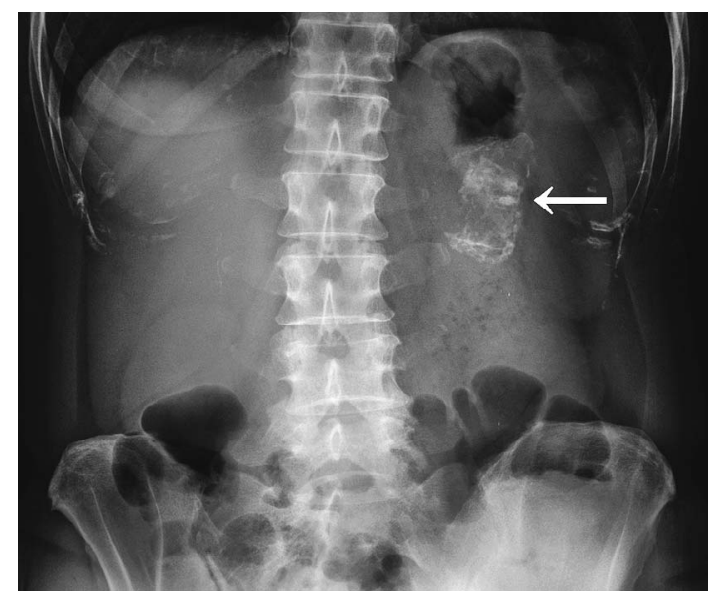

Figure 2. Plain x-ray of the abdomen showing a cloud-like calcified shadow (arrow) in the left upper quadrant of the abdomen.

the gastric body. Since the tortuous and nodular elevation was slightly bluish in color, it was suspected to be gastric varices $\left(\mathrm{Lg}-\mathrm{b}, \mathrm{F}_{2}, \mathrm{Cw}, \mathrm{RC}_{0}\right)($ Fig. 1). A plain $\mathrm{X}$-ray film of the abdomen showed a cloud-like calcified shadow in the upper right abdomen (Fig. 2). Abdominal ultrasound showed a low echoic mass with calcification in the splenic hilum (Fig. 3). Abdominal computed tomography (CT) identified a low density mass measuring about $5 \mathrm{~cm}$ in diameter near the tail of the pancreas and the splenic hilum, along with calcification and a collateral vein around the area (Fig. 4A, B and C). Three-dimensional (3D)-CT angiography (volume rendering method) displayed occlusion of the splenic vein at the pancreatic tail by the mass, with a welldeveloped collateral vein (Fig. 4D). On T1- and T2weighted MRI, a mixture of high and low signals was detected within the mass, suggestive of old and new bleeding inside a cyst (Fig. 5A and B). Based on the results of these investigations, the tumor was diagnosed as SPN, and the gastric varices were considered to have developed secondary

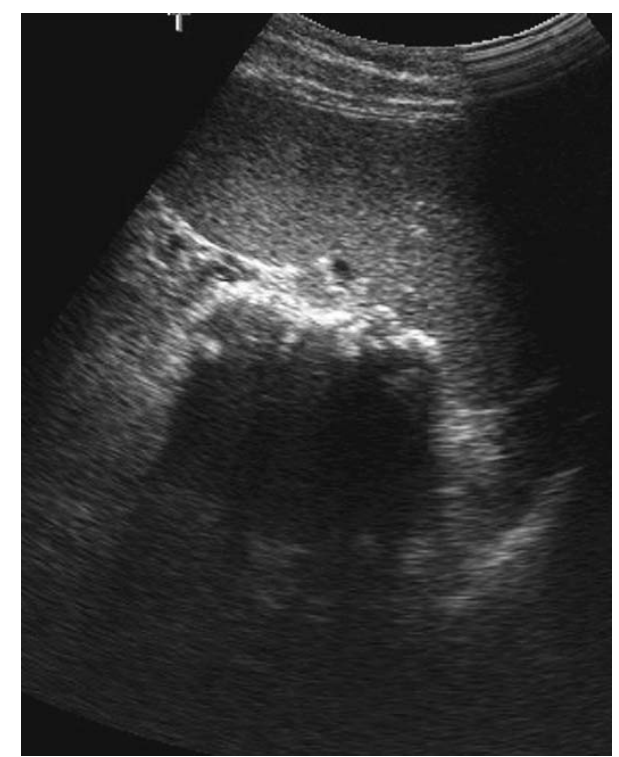

Figure 3. Abdominal ultrasonography showed dense peripheral rim calcification with posterior acoustic shadowing in the splenic hilum.

to the occlusion of the splenic vein. Distal pancreatectomy and splenectomy were performed simultaneously. The pathological findings included a congested and calcified 5-cm diameter encapsulated tumor located in the pancreatic tail. The splenic vein was occluded near the hilum of the spleen, together with several markedly developed collateral veins and splenomegaly. Examination of the resected specimen showed a solid tumor extending from the body to the tail of the pancreas. The tumor contained calcified, hemorrhagic, and necrotic areas. The splenic vein was occluded at the region of inflammation, and collateral veins were observed (Fig. 6). Histopathological findings included degenerative necrosis, the presence of cells with round nuclei that showed pseudopapillary growth (Fig. 7A). The diagnosis of SPN was confirmed based on the accumulation of nuclear $\beta$-catenin (Fig. 7B). The tumor findings were: CD10 (+/-), synaptophysin (-), chromogranin A (-), trypsin (-), Ki-67<1\%, and p 53 overexpression (-). The splenic vein was occluded by inflammation with a hyaline stroma (Fig. 7C). No vascular invasion or infiltrative growth was observed. The tumor margin was negative, and low-grade pancreatic intraepithelial neoplasia was observed in the peripancreatic parenchyma. No malignancy was found in the lymph nodes or the spleen.

\section{Discussion}

SPN, which was first reported in 1959 by Frantz, (9) is described as a rare epithelial tumor of the pancreas that accounts for $1-2 \%$ of non-endocrine pancreatic tumors. It is frequently observed in young people and women, and is usually asymptomatic, and only detected after growing as large as 2.5 to $10 \mathrm{~cm}$ in diameter. It affects the head, body and tail of the pancreas with the same frequency. Macroscopically, SPN is a round tumor with a thick fibrous cap- 

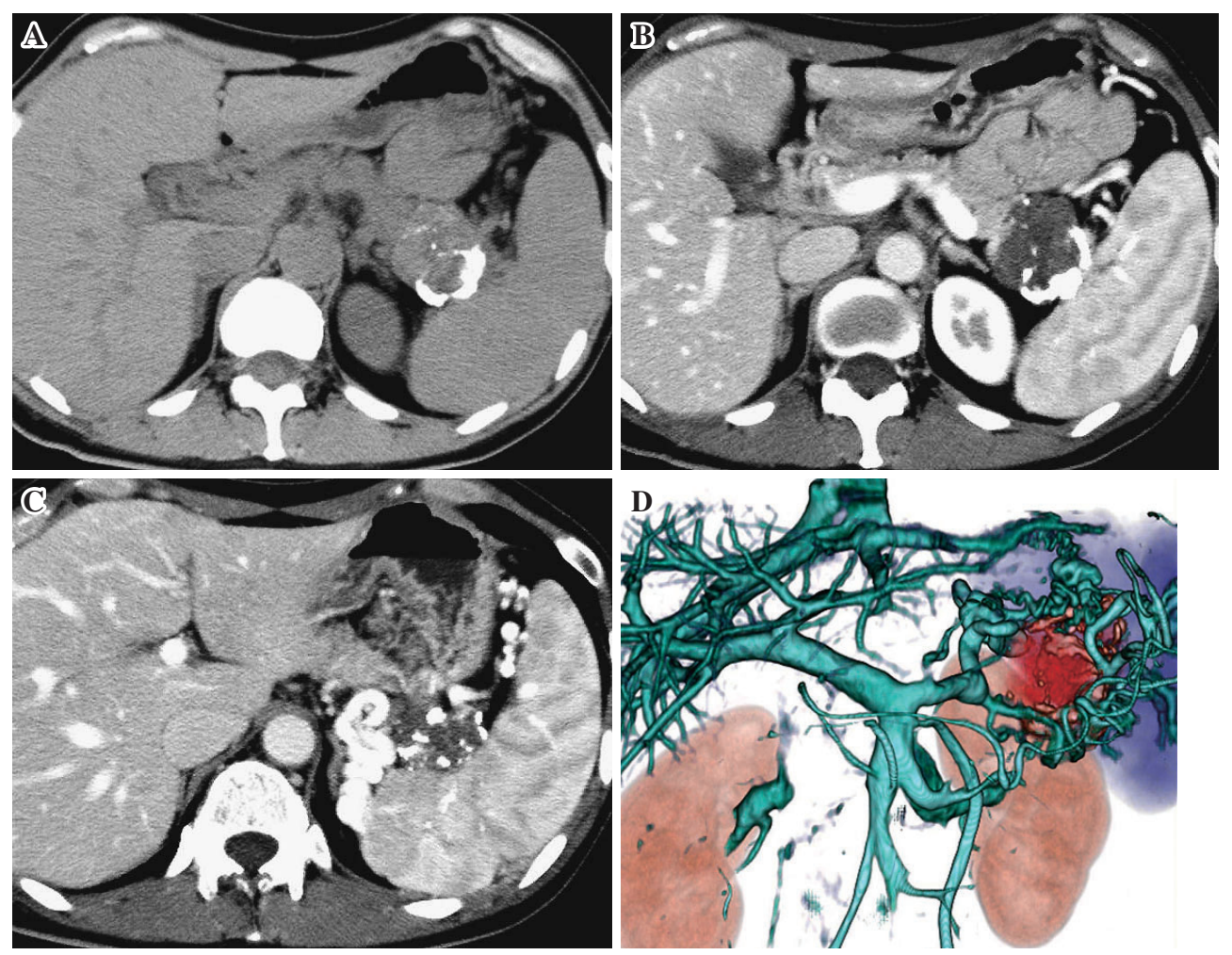

Figure 4. Abdominal computed tomography (CT) showed a low-density mass measuring about 5 $\mathrm{cm}$ in diameter near the tail of the pancreas and the splenic hilum, along with calcification. (A) Unenhanced CT. (B) Contrast-enhanced CT. (C) Contrast-enhanced CT showed a collateral vein around the tail of the pancreas and the splenic hilum. (D) 3D-CT angiography (volume rendering method) displayed occlusion of the splenic vein at the pancreatic tail by the mass, with a well-developed collateral vein.
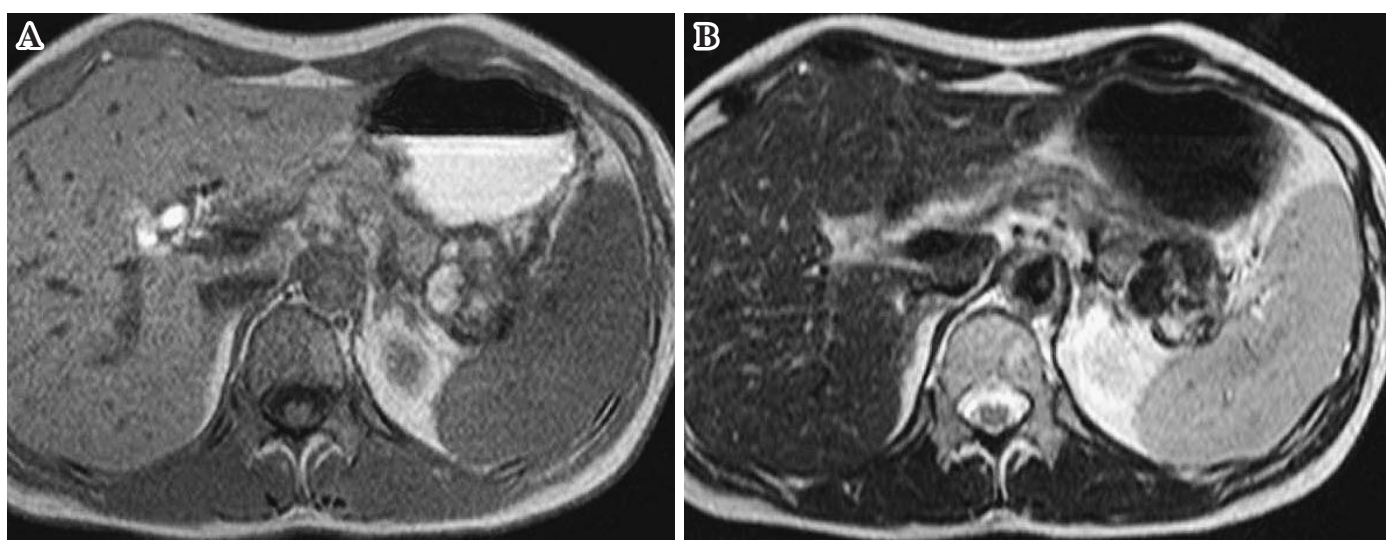

Figure 5. (A) T1-weighted magnetic resonance (MR) images showed a well-defined heterogeneous mass with a rim of low signal intensity in the tail of the pancreas. (B) T2-weighted MR images showed a well-defined heterogeneous mass in the tail of the pancreas.

sule that grows outside the pancreas, and the cut surface shows a mixture of solid parts and necrotic or hemorrhagic cysts. Calcification is also observed in the capsule and the solid parts. Histologically, the tumor displays solid growth with hyaline stroma of acidophilic cells containing small round nuclei, while pseudopapillary or pseudorosette patterns are formed around vessels. Accumulation of $\beta$-catenin is found in the nuclei of tumor cells. Almost all SPN harbor somatic point mutations in exon 3 of the $\beta$-catenin gene.
These mutations inactivate the glycogen synthase kinase-3 $\beta$ (GSK-3 $\beta$ ) phosphorylation site, and thereby interfere with the normal ubiquitin-mediated degradation of the $\beta$-catenin protein. The $\beta$-catenin protein then abnormally accumulates in the cytoplasm of these cells and is translocated to the nucleus, where it can stimulate transcription of a variety of genes including $c$-myc and cyclin D1. Accordingly, over 90 percent of SPN show an abnormal pattern of immunolabeling for $\beta$-catenin protein. Normal cells in the pancreas show 
intense membranous labeling, while nuclear and cytoplasmic labeling is evident in neoplastic cells. As one would expect in a neoplasm with $\beta$-catenin activation, cyclin D1 overexpression has also been reported in the majority of SPN (10). Since most of these tumors are benign, the prognosis after excision is good (11-13).

SPN is often detected when patients present with abdominal pain or an abdominal mass. Some cases have been reported in which SPN was detected following complaints of abdominal discomfort or abdominal trauma. However, there

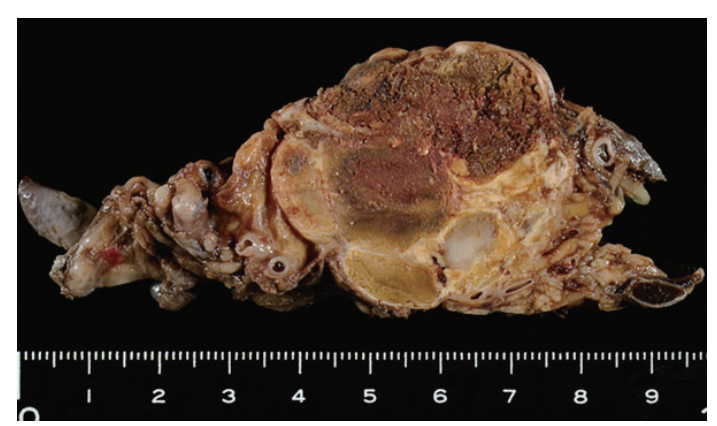

Figure 6. Photograph of the pathologic specimen showing a solid tumor measuring $5 \mathrm{~cm}$ in diameter extending from the body to the tail of the pancreas. Note the areas of calcification, hemorrhage, and necrosis in the tumor. Note also the occluded splenic vein and collateral veins. have been no reports of SPN in patients with diabetes or jaundice. Based on recent advances in imaging technology, SPN is often detected incidentally in asymptomatic patients or during follow-up of another disease. To our knowledge, the present case is the first to be reported in which SPN was detected following investigation related to gastric varices or left-sided extrahepatic portal hypertension; although a few cases of serous cystadenoma of the pancreas associated with left-sided extrahepatic portal hypertension were previously reported (6-8).

Most SPNs are benign, and there is usually less infiltration of the vascular system and surrounding tissues compared with pancreatic cancer. SPNs infrequently invade the capsule and surrounding structures, mainly the spleen, portal vein, and duodenum. Several parameters are associated with an increased potential for features of malignancy, including old age, invasion of the capsule or adjacent normal pancreatic parenchyma, as well as vascular and perineural invasion (14).

In the present case, the splenic vein was occluded by inflammation probably resulting from bleeding and necrosis associated with tumor growth. It is likely that the splenic phlebitis resulted in various compensatory changes including collateral drainage through the short gastric veins, which subsequently resulted in engorgement of the vessels along the gastric body wall and the formation of gastric varices. The present patient had a typical SPN based on both imag-

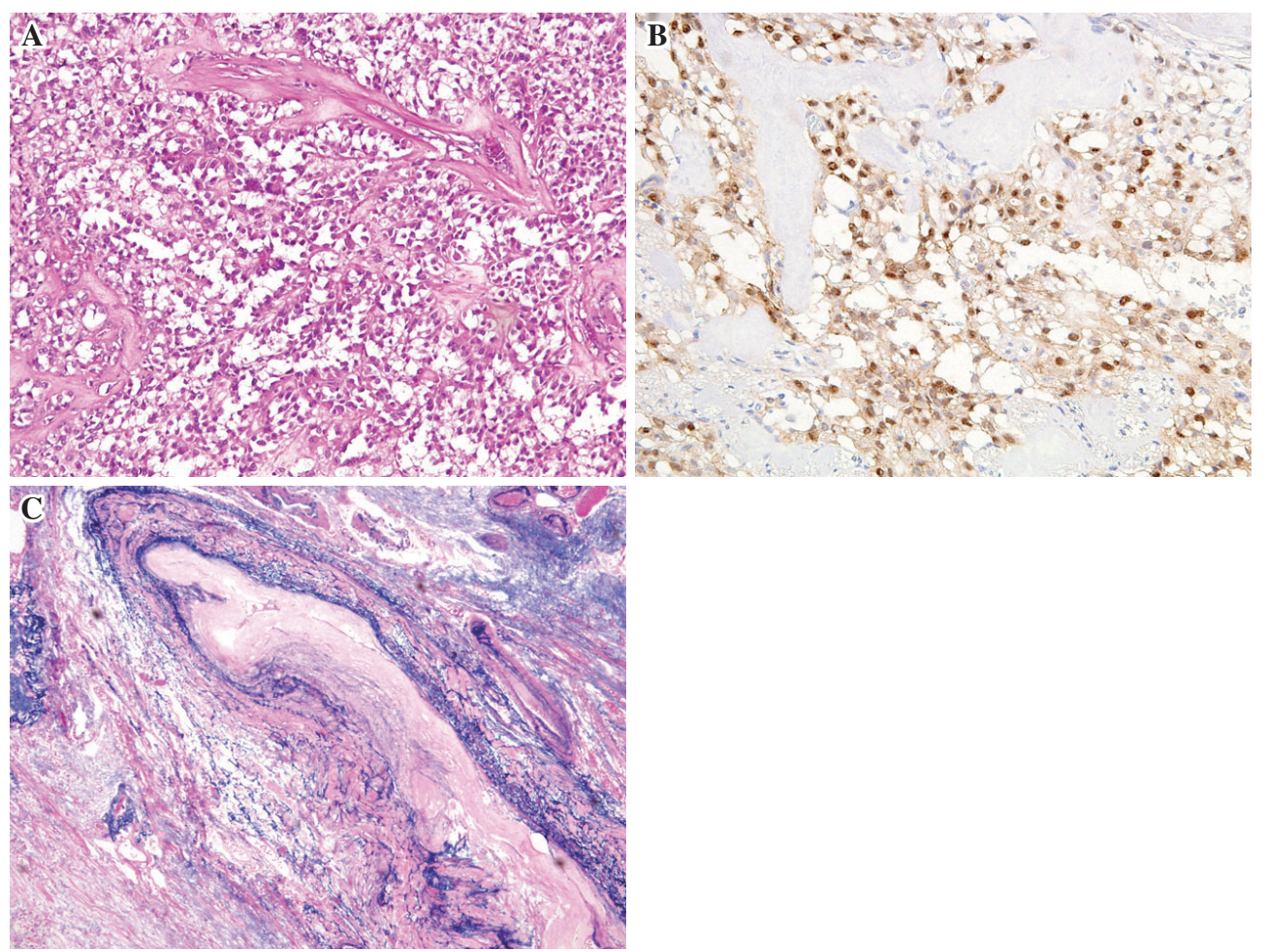

Figure 7. (A) Histopathological findings. Note the degenerative necrosis in the tumor tissue and tumor cells with round nuclei that showed pseudopapillary growth (Hematoxylin and Eosin staining, $\times 100$ ). (B) Immunohistochemical labeling for $\beta$-catenin. Note the accumulation of $\beta$-catenin in the cell nuclei $(\times 200)$. $(C)$ Occlusion of the splenic vein by inflammation with hyaline degeneration [victoria blue-Hematoxylin and Eosin $(\mathrm{VHE})$ staining. $\times 40$ ]. 
ing and pathological findings $(11,15,16)$. The CT showed a large encapsulated mass with variable solid and cystic components caused by hemorrhagic degeneration. Calcification and enhanced solid areas were also present at the periphery of the mass. In addition, 3D-CT angiography was useful for understanding the anatomy of the gastric varices and the left-sided extrahepatic portal hypertension. When varices run from the fornix to the greater curvature of the gastric body on examinations such as upper GI endoscopy, one should suspect diseases that could cause splenic vein occlusion or left-sided extrahepatic portal hypertension. This should be followed by thorough investigation to rule out any pancreatic diseases.

\section{Conclusion}

In this patient, SPN was detected during investigation of gastric varices. We described a rare case of SPN associated with splenic vein occlusion and left-sided extrahepatic portal hypertension.

\section{References}

1. Turrill FI, Mikkelsen WP. Sinistral (left-sided) extrahepatic portal hypertension. Arch Surg 99: 365-368, 1969.

2. Sutton JP, Yarborough DY, Richards JT. Isolated splenic vein occlusion. Arch Surg 100: 623-626, 1970.

3. Belli L, Puttni M, Marni A. Extrahepatic portal obstruction: clinical experience and surgical treatment in 105 patients. J Cardiovasc Surg 21: 439-448, 1980.

4. Moossa AR, Gadd MA. Isolated splenic vein thrombosis. World J Surg 9: 384-390, 1985.
5. Glynn MJ. Isolated splenic vein thrombosis. Arch Surg 121: 723725, 1986.

6. Madsen MS, Petersen TH, Sommer H. Segmental portal hypertension. Ann Surg 204: 72-77, 1986.

7. Iwasaki T, Nagata $Y$, Watahiki H, Yamamoto H, Ogawa H. A rare case of serous cystadenoma of the pancreas presenting with leftsided portal hypertension. Surg Today 26: 442-445, 1996.

8. Ito K, Kudo A, Nakamura N, Tanaka S, Teramoto K, Arii S. Leftsided portal hypertension caused by serous cystadenoma of the pancreas: Report of a case. Surg Today 38: 184-187, 2008.

9. Frantz VK. Tumors of the pancreas. In: Atlas of Tumor Pathology, 1st Series, fascicles 27 and 28. Armed Force Institute of Pathology, Washington, DC, 1959: 32-33.

10. Abraham SC, Klimstra DS, Wilentz RE, et al. Solidpseudopapillary tumors of the pancreas are genetically distinct from pancreatic ductal adenocarcinomas and almost always harbor beta-catenin mutations. Am J Pathol 160: 1361-1369, 2002.

11. Buetow PC, Buck JL, Pantongrag-Brown L, Beck KG, Ros PR, Adair CF. Solid and pseudopapillary epithelial neoplasm of the pancreas: imaging-pathologic correlation on 56 cases. Radiology 199: 707-711, 1996.

12. Coleman V, Doherty MC, Bigler SA. Solid-pseudopapillary tumor of the pancreas. Radio-Graphics 23: 1644-1648, 2003.

13. Canzonieri V, Berretta M, Buonadonna A, et al. Solid pseudopapillary tumour of the pancreas. Lancet Oncol 4: 255-256, 2003.

14. Nishihara K, Nagoshi M, Tsuneyoshi M, Yamaguchi K, Hayashi I. Papillary cystic tumors of the pancreas: assessment of their malignant potential. Cancer 71: 82-92, 1993.

15. Cantisani V, Mortele KJ, Levy A, et al. MR imaging features of solid pseudopapillary tumor of the pancreas in adult and pediatric patients. AJR 181: 395-401, 2003.

16. Choi JY, Kim MJ, Kim JH, et al. Solid pseudopapillary tumor of the pancreas: typical and atypical manifestations. AJR 187: 178186, 2006.

(C) 2010 The Japanese Society of Internal Medicine http://www.naika.or.jp/imindex.html 